\title{
Image Processing Algorithm for Quantitative Characterization of Thermal Imaging Acquired During On-line Weld Monitoring
}

\author{
${ }^{1}$ N.M.Nandhitha, ${ }^{1}$ N.Manoharan, ${ }^{1}$ B.Sheela Rani, ${ }^{2} B$.Venkatraman, ${ }^{2}$ P.Kalyana Sundaram, ${ }^{2}$ Baldev raj \\ 'Sathyabama University, Jeppiaar Nagar, Old Mamallapuram Road, Chennai 600 119, India \\ ${ }^{2}$ Indira Gandhi Centre for Atomic Research (IGCAR), Kalpakkam 603 102, India \\ E-mail: nandhi_n_m@yahoo.co.in
}

\begin{abstract}
Defect in welded structures is a matter of serious concern. The current practice involves interpretation by inspectors and experts, which is time consuming. With greater emphasis on automation during manufacturing process, automated NonDestructive Testing (NDT) Techniques have gained prominence. The key to success of automated NDT lies in the automatic defect recognition and characterization. Dimensional Characterization of defects or discontinuities is essential in order to compare this with the acceptable codes and standards. Greater emphasis is on selecting appropriate mathematical tools so that feature extraction is possible in accurate and reliable manner. Conventionally image-processing algorithms are applied to extract and quantify the features depicting defects. However many image processing algorithms are available for specific applications with its advantages and disadvantages. This paper explores the possibility of image processing algorithms like edge detection and morphological operators for feature extraction and proposes an algorithm for quantitative measurements of defects on thermal images. Lack of Fusion and Tungsten Inclusion are the defects considered. The choice of these particular defects is that these defects are the main causes for rejection and can be detected reliably during thermal imaging.
\end{abstract}

Key words : Lack of fusion, tungsten inclusion, edge, dilation, region filling and erosion

\section{INTRODUCTION}

Welding is one of the widely used methods for joining metals. In spite of the numerous advances in the science and technology of welding, failures do occur and weld is still considered to be the weakest portion. This is because the formation of the weld is affected by a number of process parameters, which make it difficult to ensure the quality of the weld. NDE techniques are used for the evaluation of weld defects. Non-destructive testing has been defined as comprising those test methods used to examine a part or material or system without impairing its future usefulness [1]. Various signal and image processing techniques are used for qualitative and quantitative characterization of the defects.

Infrared thermography is the widely used method for Non-destructive testing. Thermography offers noncontact, wide area detection of subsurface defects. Different passive and active thermographic techniques are used for defect detection. Active techniques include pulse thermography, lockin thermography, pulsed phase thermography and vibrothermography [2-4]. Numerous groups worldwide have used Infrared investigation techniques in the inspection of subsurface defects and features, thermo physical properties, coating thickness and hidden structures. Thermographs are used to the control of welding process problems, such as arc misalignments [5]. Infrared sensors are used for on-line weld geometry monitoring and control. Variations in plate thickness, shielding gas composition and minor element content causes weld geometry changes. These parameter deviations are detected from temperature gradients obtained from infrared data [6]. Infrared sensors are best suited for weld quality detection as the perturbations that arise due to variations in arc positioning, heat input and the presence of contaminants distinctly manifests itself as differences in the spatial and temporal surface temperature distributions. Hence image analysis techniques can be developed to quantify the changes in the temperature distribution there by enabling adaptive welding techniques for automated weld control [7]. Weld defects are detected and quantified from thermographs. Quantification of thermographs is achieved by image processing algorithm through histogram equalization, image segmentation and morphological image processing. These features extracted from the algorithm are then used for on-line weld monitoring to produce defect free welds [8].

This paper presents an image-processing algorithm for both qualitative and quantitative characterization of defects in weld thermographs. The major axis length, minor axis length and area are the feature vectors used for the dimensional characterization of the defects.

\section{WELD DEFECT THERMOGRAPHS}

Welding is a process associated with high temperatures and as a result strong spatial temperature gradients occur at the weld pool. During the welding process, the high temperatures associated with the arc and appropriate thermophysical properties such as thermal diffusivity cause strong spatial temperature 
gradients to occur in the weld pool. Convection in the weld pool, shape of the weld pool and heat transfer in both the solid and liquid metal determines the temperature distributions in thermal maps. The thermal maps produced by infrared thermal imaging instruments are called thermographs. Thermograph is defined as a $2 \mathrm{D}$ radiance function $g(x, y)$, where $x$ and $y$ denote spatial coordinates and the value of $g$ at any point is proportional to the radiance or energy emitted from the scene at that location. For an ideal weld with constant conditions, thermographs show repeatable and regular patterns. Perturbations in welding penetration clearly manifest itself in hot spots of the thermographs. Improper welding parameters, improper base metal preparation and wrong welding procedures introduce defects or faults in the weld metal. The common weld defects that are considered are discussed below along with their thermographs.

\section{Lack of fusion}

Sometimes the molten metal deposited by the electrodes does not fuse properly with the cold metal base and the two do not unite properly and completely. It may be due to lower arc current or faster arc travel speed or improper weaving technique. Lack of fusion appears as a region of asymmetric temperature distribution in the thermograph as shown in Fig. 1.

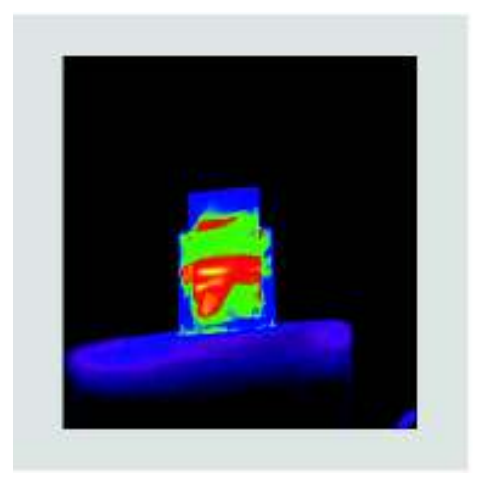

Fig. 1. Lack of fusion thermograph

\section{Tungsten inclusion}

Tungsten Inclusion is the most commonly occurring defect in GTAW welding. It occurs when a very high welding current is used for the electrode. It also occurs when the arc is unstable or when the electrode momentarily touches the weld pool. Tungsten Inclusion appears as an abrupt high temperature region in thermograph as shown in Fig. 2.

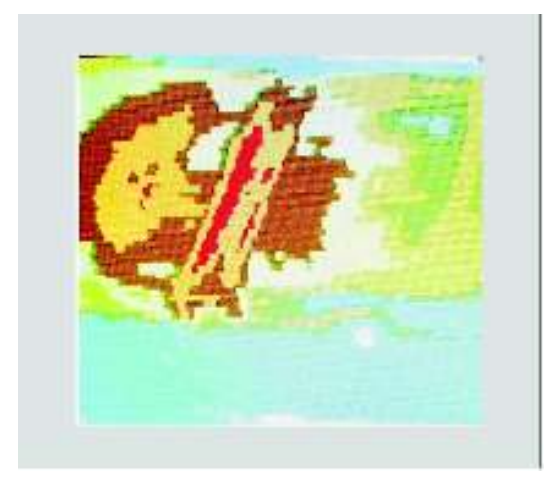

Fig.2. Tungsten inclusion thermograph

The thermographs are obtained from thermographs database maintained at IGCAR, Kalpakkam.

\section{DIGITAL IMAGE PROCESSING- A REVIEW}

An image is defined as a two-dimensional function, $f(x, y)$ where $x$ and $y$ are spatial co-ordinates, and the amplitude of $f$ at any pair of co-ordinates $(x, y)$ is called the intensity or gray level of the image at that point. When $x, y$, and the amplitude values of $f$ are all finite and discrete, the image is called a digital image. Adigital image is composed of a finite number of elements, each of which has a particular location and value. These elements are referred to as pixels. Digital image processing aims at extracting and quantifying the region of interest from the original image. The various steps involved in digital image processing are as follows.

\section{Image preprocessing}

After acquiring the thermographs, image preprocessing is performed on the images. Basically the idea of pre-processing is to improve the quality of the image so that further processing on those images will be easier and also provides better results. In our work, pre-processing involves the conversion of colored image into gray scale image and improving the gray level resolution.

\section{Image segmentation}

Segmentation subdivides an image into its constituent regions or objects. The level to which the subdivision is carried out depends on the problem being solved. That is, segmentation should stop when the objects of interest in an application have been isolated. Image segmentation algorithms are usually based on one of two basic properties of intensity values namely discontinuity and similarity. In this work, segmentation is achieved through edge detection. An edge is defined as a set of connected pixels that lie on the boundary between two different gray levels. A pint in an image can be 
regarded as a edge pixel if its two-dimensional first order derivative is greater than a specified threshold. First order derivatives of a digital image are based on various approximations of the $2 \mathrm{D}$ gradient. The most commonly used gradient operators are described below.

Let the $3 \times 3$ area shown in the Fig. 3 represent the gray levels in the neighborhood of the image.

\begin{tabular}{|l|l|l|}
\hline$Z_{1}$ & $Z_{2}$ & $Z_{3}$ \\
\hline$Z_{4}$ & $Z_{5}$ & $Z_{6}$ \\
\hline$Z_{7}$ & $Z_{\text {B }}$ & $Z_{9}$ \\
\hline
\end{tabular}

Fig. $3.3 \times 3$ Neighborhood of image

Let $G_{x}$ and $G_{y}$ represent the partial derivatives of gray level along $x$ and $y$ directions. Then

$$
G_{x}=\left(z_{7}+z_{8}+z_{9}\right)-\left(z_{1}+z_{2}+z_{3}\right)
$$

and

$\mathrm{G}_{\mathrm{y}}=\left(\mathrm{z}_{3}+\mathrm{z}_{5}+\mathrm{z}_{9}\right)-\left(\mathrm{z}_{1}+\mathrm{z}_{4}+\mathrm{z}_{7}\right)$

These equations are proposed by Prewitt A slight variation of these two equations as proposed by Sobel [9]. They are

$$
\begin{gathered}
G_{x}=\left(z_{7}+2 z_{8}+z_{9}\right)-\left(z_{1}+2 z_{2}+z_{3}\right) \\
\text { and } \\
G_{y}=\left(z_{3}+2 z_{5}+z_{9}\right)-\left(z_{1}+2 z_{4}+z_{7}\right)
\end{gathered}
$$

\section{Morphological image processing}

Mathematical morphology is for extracting image components that are useful in the representation and description of region shape such as boundaries, skeletons etc. It involves dilation, region filling and erosion. Dilation is used for bridging the gap between the pixels in an image. Erosion aims at removing the irrelevant details in an image. Region filling makes all the pixels in the bounded regions as white thereby differentiating it from the background. The success of the above morphological operations is dependent on the chosen structuring elements.

\section{Representation and description}

Once the region of interest (defect) is obtained from the above process, it has to be represented and described for interpretation. Here the prime focus is on the size of the defect. Hence external descriptors are used for characterizing the defect. Major axis length, Minor axis length and area are the feature vectors used for the quantitative characterization of the defects.

\section{WELD FEATURE EXTRACTION}

The various steps involved in weld feature extraction and quantification is as described below.

Step 1 Acquired thermographs are converted from color domain to gray scale. Performing operations in color domain leads to computational complexity as enhancement and edge detection operations are to be dealt with RGB component vectors. Moreover success of color image processing is also dependent on the chosen color models. Hence it is necessary to convert the image to gray scale.

Step 2 The gray level resolution is increased by representing each pixel with 16 bits instead of 8 bits. Gray level resolution refers to the smallest discernible change in gray level. Normally each pixel in gray scale is represented with 8 bits resulting in 256 quantization levels. So accuracy of information is achieved by increasing the number of bits used to represent the pixel as 16 , which increases the number of discrete gray levels.

Step 3 Edge detection is performed by choosing appropriate filter and threshold. In this work, edge detection is performed with Sobel masks as they have slightly superior noise-suppression characteristics [9]. The $3 \times 3$ Sobel masks used for detecting edges along $x$ and $y$ directions are as shown in Fig.4. and Fig. 5.

\begin{tabular}{|l|l|l|}
\hline-1 & 0 & 1 \\
\hline-2 & 0 & 2 \\
\hline-1 & 0 & 1 \\
\hline
\end{tabular}

Fig. 4. Vertical mask

\begin{tabular}{|c|c|c|}
\hline-1 & -2 & -1 \\
\hline 0 & 0 & 0 \\
\hline 1 & 2 & 1 \\
\hline
\end{tabular}

Fig. 5. Horizontal mask

Step 4 Dilation is done on the edge-detected image. It bridges the gap between the edge pixels. The structuring elements chosen are horizontal and vertical lines. The dilated image is then region filled with holes as the structuring elements. The region filled image is then eroded. The structuring element chosen is diamond of 1 pixel size. 
Step 5 Major axis length, minor axis length and area of the defect are obtained. These feature vectors provide the quantitative characterization of the defects.

\section{RESULTS AND DISCUSSION}

The resultant thermographs at various stages of the image processing algorithm for Lack of Fusion is as shown in the Fig. 6.

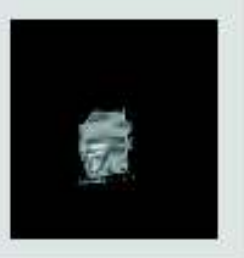

Fig.6 a. Gray scale image

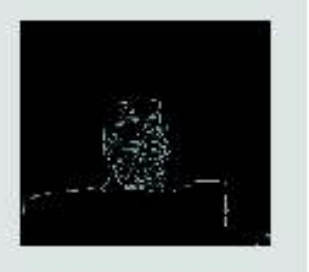

Fig. 6b. Edge detected image

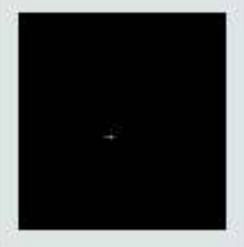

Fig.6c. Defect isolated image (lack of fusion)

Similarly the thermograph outputs at various stages of the algorithm for Tungsten Inclusion are as shown in the Fig. 7.
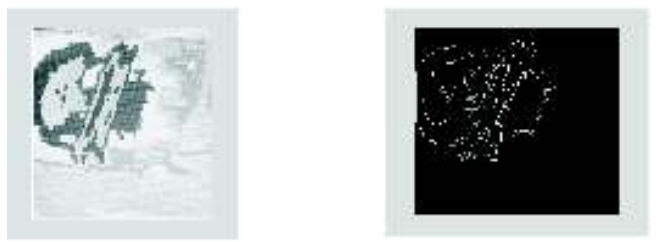

Fig.7a. Gray scale image Fig. 7b. Edge detected image

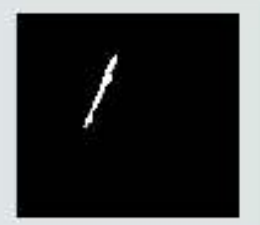

Fig.7c. Defect Isolated image (Lack of Fusion)

The dimensional characterization of the defects is as shown in the Table 1.
Table 1. Quantitative characterization of defect

\begin{tabular}{|l|l|l|}
\hline $\begin{array}{l}\text { Defect/Feature } \\
\text { Vector }\end{array}$ & $\begin{array}{l}\text { Lack of } \\
\text { Fusion }\end{array}$ & $\begin{array}{l}\text { Tungsten } \\
\text { Inclusion }\end{array}$ \\
\hline $\begin{array}{l}\text { Major Axis Length } \\
\text { (pixels) }\end{array}$ & 46.79 & 137.0795 \\
\hline $\begin{array}{l}\text { Minor Axis Length } \\
\text { (pixels) }\end{array}$ & 6.09 & 13.1303 \\
\hline Area (Pixels) & 218 & 1227 \\
\hline $\begin{array}{l}\text { Major Axis Length } \\
\text { (in cm.) }\end{array}$ & 1.4 & 4.1123 \\
\hline $\begin{array}{l}\text { Minor Axis Length } \\
\text { (in cm.) }\end{array}$ & 0.18 & 0.3939 \\
\hline Area (in sq. cm) & 0.1962 & 1.1043 \\
\hline
\end{tabular}

The algorithm automatically detects and quantifies the defects. Major axis length, minor axis length and area are used to quantify the size of these defects.

\section{CONCLUSION}

An automated defect detection and quantification algorithm is developed to characterize the defect. Proper threshold and structuring elements are chosen for effective defect detection. These algorithms effectively isolate defects and quantify the defects by obtaining the major axis length, minor axis length and area of the defect. These parameters are defined in pixels.

In the proposed method, success of image segmentation is dependent on the threshold set for edge detection. Morphological image processing is also dependent on the choice and size of the structuring elements. The effectiveness of this algorithm is hence dependent on various parameters chosen for different operations. In order to develop a better system, image dependency and parameter dependency are to be avoided.

\section{REFERENCES}

[1] Non Destructive Testing Handbook, Second edition: Vol. 10, Non Destructive Testing Overview. Columbus, OH: American Society for Non Destructive Testing (1996)

[2] Khan.M.A, Madsen. N.H., Goodling. G.S. and Chin. B.A., 1986, Infrared Thermography as a Control for the Welding Process, optical Engineering, Vol.25, pp.799-805.

[3] Banarjee. P., Govardhan. S., Wikle. H.C., Liu. J.Y. and Chin. B.A., 1995, Infrared Sensing for On-Line Weld Geometry Monitoring and Control, Journal of Engineering for Industry, Vol.117, pp.323-330. 
[4] Ibarra-Castanedo. C., Galmiche. F., Darabi. A., Pilla. M., Klein. M., Ziadi. A., Vallerand. S., Pelletier. J.-F., Maldague. X., 2003, Thermographic nondestructive evaluation: overview of recent progress, SPIE Proc. Thermosense XXV (SPIE: Society of Photo-Optical Instrumentation Engineers), 5073: 450-459, Orlando, X. Maldague, A. Rozlosnikeds., FL.,

[5] Maldague. X., 2002, Introduction to NDT by Active Infrared Thermography, Materials Evaluation, 6[9]: 10601073.

[6] Maldague. X., 2000, Applications of Infrared Thermography in NonDestructive Evaluation,
Trends in Optical Nondestructive Testing (invited chapter), Pramod Rastogi ed., pp. 591-609.

[7] Nagarajan, S., Chen. W.H. and Chin. B.A., 1989, Infrared Sensing for Adaptive Arc Welding", Welding Journal, Vol.68, pp.462s-466s.

[8] Venkataraman. B., Baldev Raj and Menaka. M., 2005, "Online Infrared Detection of Inclusions and Lack of Penetration during Welding", Materials Evaluation, pp. 933-937.

[9] Chanda B., Dutta D. Majumder, 2002, "Digital Image Processing and Analysis", Prentice Hall of India. 